\title{
Miniaturized Tables of Bessel Functions. III*
}

\author{
By Yudell L. Luke
}

\begin{abstract}
After the manner of our previous studies, coefficients for the expansion of $J_{v}(z)$ and $Y_{v}(z)$ in double series of Chebyshev polynomials are presented. For $J_{v}(z)$, the ranges are (1) $0<z \leqq 8,0 \leqq \nu \leqq 4,(2) 0<z \leqq 8,4 \leqq \nu \leqq 8$. For $J_{\nu}(z)+i Y_{\nu}(z)$, the ranges are $z \geqq 5$ and $0 \leqq \nu \leqq 1$. The coefficients are given with sufficient accuracy to enable the evaluation of the Bessel functions to at least 20 decimals.
\end{abstract}

1. Introduction. In previous studies [1], [2], we considered the expansion of two parameter functions in a double series of Chebyshev polynomials and developed coefficients for the evaluation of $K_{\nu}(z)$ and $I_{\nu}(z)$ over a large part of the real $z$ and $\nu$ lines. In the present paper, we give similar type coefficients for the evaluation of $J_{\nu}(z)$ and $Y_{\nu}(z)$.

2. Chebyshev Expansion for $J_{v}(z)$. From [3, Vol. 1, p. 212 and Vol. 2, p. 35],

$$
\begin{gathered}
J_{\nu}(z)=z^{\prime} \sum_{k=0}^{\infty} A_{k}(\nu, \lambda) T_{2 k}(z / \lambda), \quad 0<z \leqq \lambda, \\
A_{k}(\nu, \lambda)=G_{k}(\nu, \lambda) / 2^{\nu} \Gamma(\nu+1),
\end{gathered}
$$

$$
G_{k}(\nu, \lambda)=\frac{\epsilon_{k}(-)^{k} \lambda^{2 k} \Gamma(\nu+1)}{2^{4 k} k ! \Gamma(k+\nu+1)}{ }_{1} F_{2}\left(\begin{array}{c}
\frac{1}{2}+k \\
1+2 k, \nu+1+k
\end{array} \mid-\lambda^{2} / 4\right),
$$

$\frac{2 G_{k}(\nu, \lambda)}{\epsilon_{k}}=\frac{(k+1)}{(k+2)}\left\{G_{k+1}(\nu, \lambda)-G_{k+3}(\nu, \lambda)\right\}-\frac{16(k+1)(k+\nu+1)}{\lambda^{2}} G_{k+1}(\nu, \lambda)$

$$
+\left\{1-\frac{16(k+1)(k+2-\nu)}{\lambda^{2}}\right\} G_{k+2}(\nu, \lambda),
$$

where

$$
\epsilon_{0}=1, \quad \epsilon_{k}=2 \text { for } k>0 .
$$

It is readily shown that

$$
G_{k}(\nu, \lambda)=\frac{\epsilon_{k}(-)^{k} \lambda^{2 k} k^{-\nu}}{2^{1 k}(k !)^{2}}\left[1+O\left(k^{-1}\right)\right]
$$

and for $\nu$ and $\lambda$ fixed,

Received July $18,1971$.

AMS 1969 subject classifications. Primary 3325, 4216, 6505, 6525.

Key words and phrases. Bessel functions, approximation of bivariate functions, expansions in double series of Chebyshev polynomials, mathematical tables.

* This research was supported by the United States Atomic Energy Commission under Contract AT(11-1) 1619. 


$$
\lim _{k \rightarrow \infty} G_{k}(\nu, \lambda)=0 .
$$

Thus, the expansion (1) converges and by letting $z \rightarrow 0$, we have the useful normalization relation

$$
\sum_{k=0}^{\infty}(-)^{k} A_{k}(\nu, \lambda)=1
$$

Further, after the manner of the discussion presented in [3, Vol. 2, pp. 159-166], we can show that use of the recursion formula (4) in the backward direction is convergent. Thus, for a fixed $\lambda$, we can generate the coefficients $A_{k}(\nu, \lambda)$ for any given value of $\nu$. Then, following the discussion given in [1], we can develop coefficients $D_{r, k}(\lambda)$ such that

$$
A_{k}(\nu, \lambda)=\sum_{r=0}^{\infty} D_{r, k}(\lambda) T_{r}^{*}\left(\frac{\nu-s}{t}\right), \quad s \leqq \nu \leqq s+t .
$$

We remark that 20 decimal values of $A_{k}(\nu, \lambda)$ are given in [3, pp. 331, 332, 352-356] for $\lambda=8$ and $\nu=0, \pm \frac{1}{4}, \pm \frac{1}{3}, \pm \frac{1}{2}, \pm \frac{2}{3}, \pm \frac{3}{4}, 1$. Coefficients for the evaluation of $Y_{0}(z)$ and $Y_{1}(z)$ for $0<z \leqq 8$ are also given in [3, pp. 331, 332].

We next present a descending type expansion in series of Chebyshev polynomials for the evaluation of $J_{\nu}(z)$ and $Y_{\nu}(z)$ in the vicinity of $z=+\infty$. Now,

$$
H_{\nu}^{(1)}(z)=-\frac{2 i}{\pi} e^{-i \nu \pi / 2} K_{\nu}\left(z e^{-i \pi / 2}\right)
$$

and from [1], we have

$$
K_{\nu}(z)=(\pi / 2 z)^{1 / 2} e^{-s} \sum_{k=0}^{\infty} G_{k}(\nu, \lambda) T_{k}^{*}(\lambda / z), \lambda \text { fixed, } \lambda / z \leqq 1,|\arg z|<3 \pi / 2
$$

The recursion formula for $G_{k}(\nu, \lambda)$ and other properties of these coefficients are given in [1]. If we write

$$
\begin{aligned}
H_{\nu}^{(1)}(z) & =J_{\nu}(z)+i Y_{\nu}(z) \\
& =(2 / \pi z)^{1 / 2} e^{i(z-\nu \pi / 2-\pi / 4)} \sum_{k=0}^{\infty} H_{k}(\nu, \lambda) T_{k}^{*}(\lambda / z), \quad z \geqq \lambda,
\end{aligned}
$$

then the recurrence formula and other properties of the coefficients $H_{k}(\nu, \lambda)$ follow from those for $G_{k}(\nu, \lambda)$ upon replacing $\lambda$ by $\lambda e^{-i \pi / 2}$. We have the normalization relation

$$
\sum_{k=0}^{\infty}(-)^{k} H_{k}(\nu, \lambda)=1
$$

and from [1], use of the backward recurrence relation for $H_{k}(\nu, \lambda)$ is convergent provided $|\arg \lambda|<\pi / 2$. have

3. Numerical Results. From (1) and (9) with a slight change of notation, we

$$
J_{\nu}(z)=z^{\prime} \sum_{k=0}^{\infty} A_{k}(\nu) T_{2 k}(z / 8), \quad 0<z \leqq 8,
$$




$$
A_{k}(\nu)=\sum_{r=0}^{\infty} D_{r, k} T_{r}^{*}\left(\frac{\nu-s}{t}\right), \quad s \leqq \nu \leqq s+t .
$$

In Tables 1 and 2, in the microfiche section of this issue, we present values of $D_{r, k}$ which were computed by the technique depicted in [1] for $s=0, t=4$ and $s=t=4$, respectively. Values of $\Gamma(\nu+1)$ required in the numerics were obtained by use of the schema of my previous paper [4]. Numerous checks were made on the coefficients. They are of the type previously discussed in [1], [2] and we dispense with further details. The computations were designed so that the coefficients for $0 \leqq \nu \leqq 4$ are accurate to about 25D while those for $4 \leqq \nu \leqq 8$ are accurate to about 27D. To evaluate $J_{v}(z)$, we must incorporate the value of $z^{\gamma}$. As $0 \leqq z \leqq 8$, we see that the coefficients are sufficiently accurate to produce $J_{v}(z)$ to about 20 decimals at least.

Now,

$$
Y_{\nu}(z)=(\csc \nu \pi)\left[(\cos \nu \pi) J_{\nu}(z)-J_{-\nu}(z)\right]
$$

and both $J_{v}(z)$ and $Y_{v}(z)$ satisfy the same recurrence formula

$$
J_{v+1}(z)+J_{v-1}(z)=(2 v / z) J_{v}(z) .
$$

Further, the recurrence formula for $J_{v}(z)$ is always stable in the backward direction, but only conditionally stable in the forward direction. On the other hand, the recurrence formula for $Y_{v}(z)$ is always stable in the forward direction. Thus, with the aid of the coefficients just described and the recurrence formulas, we can evaluate $Y_{,}(z)$ for all $z$ such that $0 \leqq z \leqq 8$ and all $\nu>0, \nu$ an integer excepted. We have already referred to the availability of coefficients to compute $Y_{0}(z)$ and $Y_{1}(z)$. These together with the recurrence formula for $Y_{\eta}(z)$ can be used to generate values of $Y_{n}(z), n=2,3, \cdots$. As use of the recurrence formula in the forward direction for $J_{v}(z)$ is limited, we leave for a future paper the development of Chebyshev coefficients for $0 \leqq z \leqq 8$ and $\nu>8$.

Using (12) with a slight change of notation, we write

$$
J_{\nu}(z)+i Y_{\nu}(z)=(2 / \pi z)^{1 / 2} e^{i(z-\nabla x / 2-\pi / 4)} \sum_{k=0}^{\infty} H_{k}(\nu) T_{k}^{*}(5 / z), \quad z \geqq 5 .
$$

Let

$$
H_{k}(\nu)=\sum_{r=0}^{\infty} E_{r, k} T_{r}^{*}(\nu), \quad 0 \leqq \nu \leqq 1 .
$$

Table 3, also in the microfiche section of this issue, lists values of the real and imaginary parts of $E_{r, k}$. These were obtained and checked by the methods previously described and we omit further details. The coefficients are sufficiently accurate to produce values of $J_{\nu}(z)$ and $Y_{\nu}(z)$ for $\nu$ and $z$ as noted to about 25 decimals. Since

$$
\begin{aligned}
& Y_{-\nu}(z)=(\cos \nu \pi) Y_{\nu}(z)+(\sin \nu \pi) J_{\nu}(z), \\
& J_{-\nu}(z)=(\cos \nu \pi) J_{\nu}(z)-(\sin \nu \pi) Y_{\nu}(z),
\end{aligned}
$$

the coefficients $E_{r, k}$ together with the recurrence formula for $Y_{\nu}(z)$ enable the evaluation of $Y_{\nu}(z)$ for all $\nu>0$ and $z \geqq 5$. A like statement cannot be made for $J_{\nu}(z)$ as use of the recurrence formula in the forward direction for $J_{v}(z)$ is limited. We defer 
the development of coefficients to facilitate the evaluation of $J_{\nu}(z)$ when both $\nu$ and $z$ are large to a later paper.

4. Acknowledgment. I am indebted to Miss Rosemary Moran for her assistance in the numerics.

Mathematics Department

University of Missouri

Kansas City, Missouri 64110

1. Y. L. LuKe, "Miniaturized tables of Bessel functions," Math. Comp., v. 25, 1971, pp. 323-330.

2. Y. L. Luxe, "Miniaturized tables of Bessel functions. II," Math. Comp., v. 25, 1971, pp. 789-795.

3. Y. L. LuKE, The Special Functions and Their Approximations. Vols. 1, 2, Math. in Sci. and Engineering, vol. 53, Academic Press, New York, 1969. MR 39 \#3039; MR 40 \#2909. 4. Y. L. LUKE, "Evaluation of the gamma function by means of Padé approximations," SIAM J. Math. Anal., v. 1, 1970, pp. 266-281. 